Research Article

\title{
Thermal Design and Verification of Spherical Scientific Satellite Q-SAT
}

\author{
Yunhan He, Boxin Li, Zhaokui Wang $\mathbb{D}^{D}$, and Yulin Zhang \\ School of Aerospace Engineering, Tsinghua University, Beijing 100084, China \\ Correspondence should be addressed to Zhaokui Wang; wangzk@tsinghua.edu.cn
}

Received 16 March 2021; Revised 9 August 2021; Accepted 26 August 2021; Published 17 September 2021

Academic Editor: Angelo Cervone

Copyright (c) 2021 He Yunhan et al. This is an open access article distributed under the Creative Commons Attribution License, which permits unrestricted use, distribution, and reproduction in any medium, provided the original work is properly cited.

\begin{abstract}
Small satellites have gradually become an important mean of space scientific exploration. The Tsinghua University developed a spherical small satellite, Q-SAT, which is aimed at detecting the Earth gravity and atmosphere parameters. In the current paper, thermal control for Q-SAT is discussed. For heat exchange between the satellite and the environment, radiation plays the main part. Different from traditional cuboid satellites, the current spherical satellite has no individual heat input and output plane which brings challenges to the thermal design of the satellite. In addition, the cost of small satellites is required to be as low as possible. A passive thermal control solution based on integrated spherical structure is employed on the Q-SAT. The combination of two integrated hemispheres is designed to facilitate the heat conduction. Different materials are utilized to control the heat transfer path. Firstly, a set of numerical simulations demonstrate that the current design can be well adapted to complex flight environment. Next, the thermal design is verified by thermal tests. As the traditional heat radiation lamps cannot meet the test requirements of the spherical satellite, an external heat flow test method which is based on distributed heaters is proposed. Results from numerical simulations agree well with the experimental test results. Both results show that the thermal system can guarantee the functions of the satellite. Q-SAT was successfully launched into orbit on August 6, 2020. The telemetry data from Q-SAT verified the effectiveness of the satellite thermal system. The thermal design and test method proposed in present paper can potentially be adopted to other small scientific satellites as well.
\end{abstract}

\section{Introduction}

The background of this research is the small satellite developed by the Tsinghua University, Q-SAT. The mission of Q-SAT is upper atmospheric density and gravity field detection in low Earth orbit (LEO) [1]. The main payload of QSAT is a GNSS receiver, and it can determine the position of Q-SAT precisely. The precise orbit data can be used to calculate the aerodynamic drag force of Q-SAT. The atmospheric parameter detection task requires that the area-tomass ratio and aerodynamic drag coefficient of satellite have a high certainty to reduce the error of measurement. Therefore, Q-SAT is designed to be spherical to complete the mission better, which is unique to common cuboid satellites.

Thermal control is necessary for satellites. The task of the satellite thermal control system is to guarantee the temperature of the components within its proper range when the satellite is exposed to the outer space environment during the orbital mission [2, 3]. The main form of heat exchange between the satellite and the environment is radiation. The thermal characteristics of spherical satellite is different from common polyhedral ones. Therefore, the thermal control system of Q-SAT needs to be designed specifically.

Thermal design relies on active or passive temperature control devices. The active thermal control devices [4] include pumped loop systems, heaters, shape memory alloy (SMA) [5], and louvers [6]. Small satellites usually have limited size and mass, so it is difficult to use active thermal control devices widely $[2,7]$. Passive thermal control system refers to the use of the conduction and radiation characteristics of materials to autonomously control the temperature. Materials often used for passive thermal control include multilayer insulation, coatings/paints, mirror, radiating sur- 


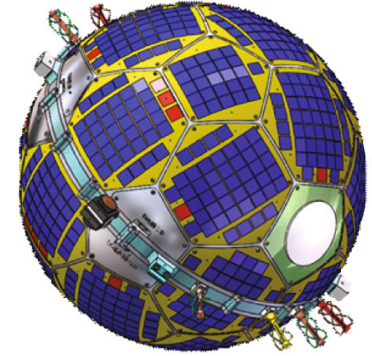

(1) Overview

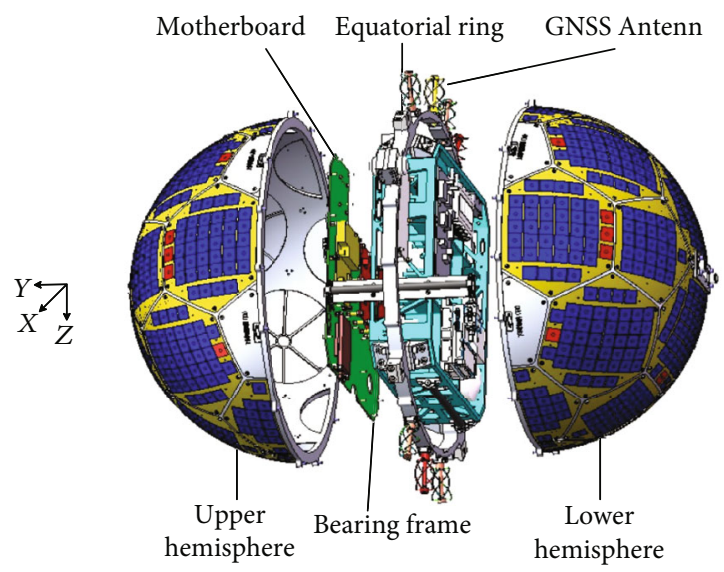

(2) Layout

Figure 1: Overview and layout of Q-SAT.

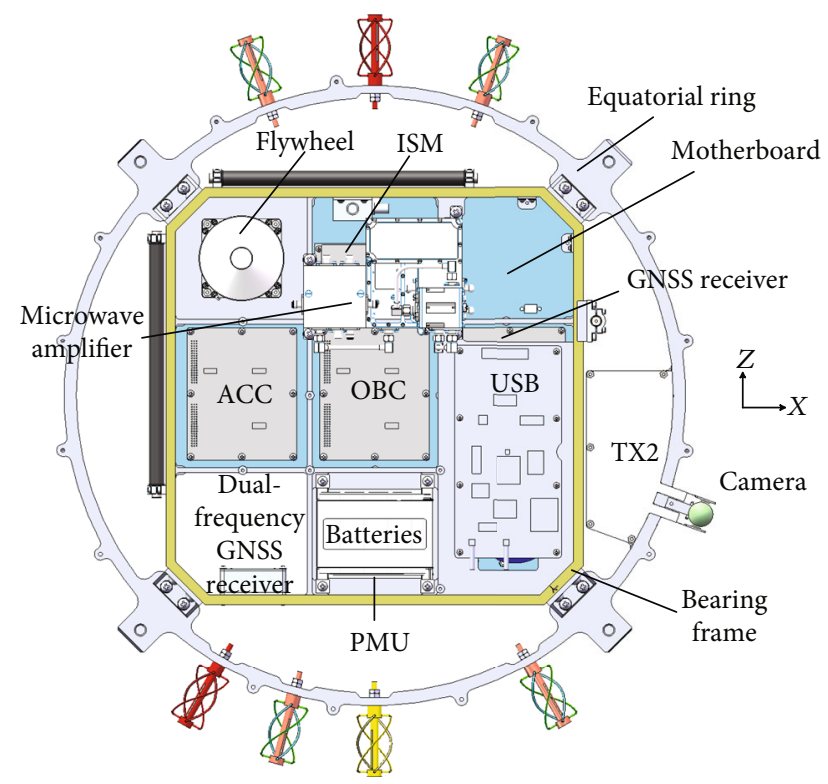

FIGURE 2: Devices distributed inside Q-SAT.

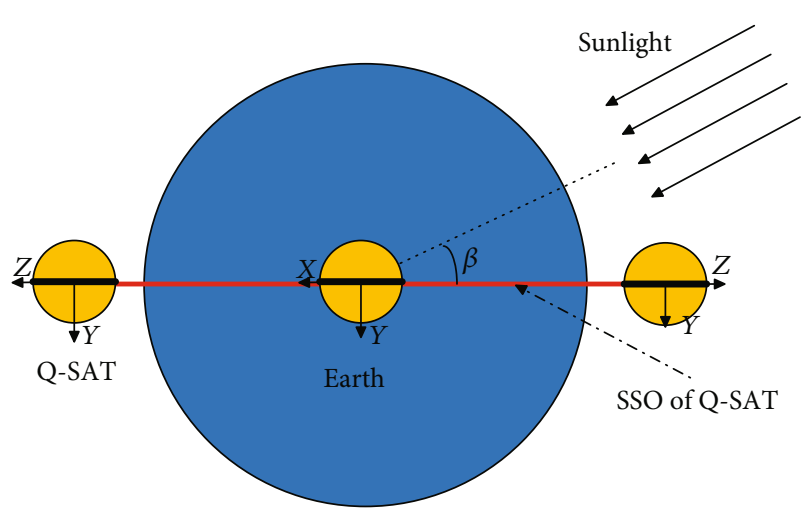

Figure 3: Orbit and condition of Q-SAT.

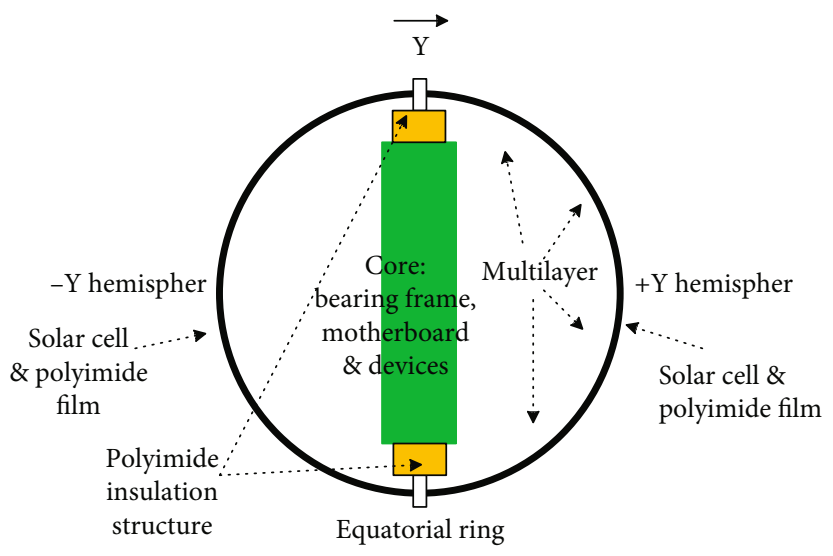

FIgUre 4: Overview of Q-SAT thermal design.

faces, thermal grease, and polyimide structure $[4,6,8-10]$. In addition, the passive thermal control system does not require an additional power source, which can save the power of the satellite [11]. More importantly, the cost of passive thermal control devices is very low. The hardware cost of the passive thermal control system applied to the small satellite can be as low as 148 dollars [12]. Therefore, the passive method is the main solution for the thermal control system of small satellites. Of course, the effectiveness of passive thermal control system depends on the accurate analysis and effective test $[11,13-15]$. The thermal design philosophy of Q-SAT is mainly a passive control system supplemented by active heaters based on the above facts.

The thermal test is a criterion for judging whether the thermal design is effective. The thermal test should accurately simulate satellite's thermal environment. The key of thermal test is to simulate the heat flow input, usually radiation from different sources. The most common method is to use radiant lamps distributed inside the vacuum chamber as the input heat source [16]. However, the heat input at different positions on the spherical surface cannot be accurately controlled when multiple radiant lamps are working at the 


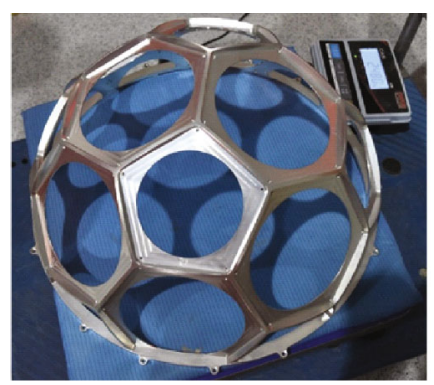

(1) Frame

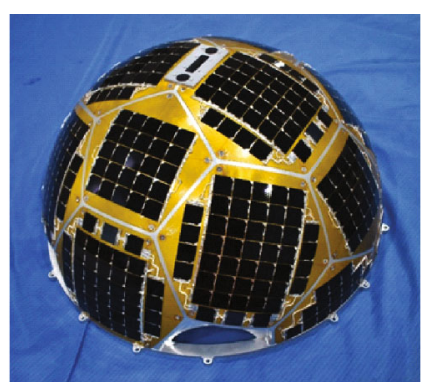

(2) With solar panels

FIgURE 5: The hemisphere.

TABLE 1: WHC and WCC parameters.

\begin{tabular}{ccccc}
\hline Case & Date & Internal heat source & $\beta$ & $\begin{array}{c}\text { Solar } \\
\text { constant }\end{array}$ \\
\hline WHC & 2021.2 .10 & $\begin{array}{c}\text { Devices run at max } \\
\text { power } \\
\text { WCC }\end{array}$ & $29.17^{\circ}$ & $1414 \mathrm{~W} / \mathrm{m}^{2}$ \\
& $\begin{array}{c}\text { Devices run at min } \\
\text { power }\end{array}$ & $19.16^{\circ}$ & $1322 \mathrm{~W} / \mathrm{m}^{2}$ \\
\hline
\end{tabular}

same time. Considering that the heat input at different positions of the sphere is not the same, a distributed external heat flow simulation method based on electric heaters of polyimide film type for the spherical satellite is proposed and tried in this mission. Another key factor in thermal testing is cost. Test expenses account for a large proportion (up to $1 / 3$ ) of satellite $R \& D$ costs [17]. The cost of thermal testing of satellites is particularly expensive because of the continuous use of liquid nitrogen on the thermal system, vacuum pumps, and cold fingers. Thermal test usually requires a large number of professionals for the inherent $24 \mathrm{~h}$ test process. Besides that, very specific kinds of materials and devices such as infrared lamps, electrical cables, electrical heaters, tapes, and films are required to comprise the test setup [18]. Considering the above factors, the thermal test is simplified from the following aspects: (a) test focuses on the key devices of the small satellite as a whole to lower the cost instead of testing devices one by one, and there is no need to conduct specialized verification for devices with flight experience. (b) The thermal test is conducted in a strategic sequence.

The second section of the article introduces the Q-SAT structure and thermal control design solution. The simulation results are given. The third section reports the methods and results of the thermal test. The fourth section shows the actual temperature state of the satellite flying on orbit. The conclusion is given in the last section.

\section{Thermal Design}

2.1. Structure of Q-SAT. The overview and the layout of QSAT are shown in Figure 1. The outer surface of the satellite is a sealed sphere with a diameter of $510 \mathrm{~mm}$. Solar cells are mounted on the surface of Q-SAT to keep the spherical shape. The structure of the satellite includes two hemispher-

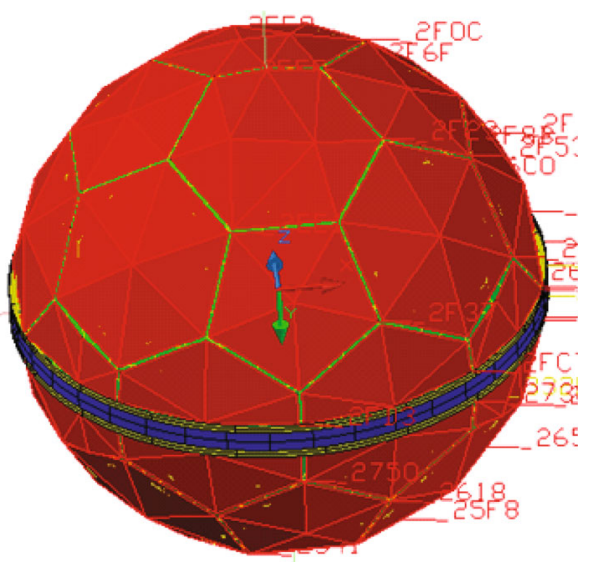

Figure 6: Thermal model of Q-SAT.

ical shells, an equatorial bearing ring, a bearing frame, and a motherboard. The two hemispherical shells are fixedly connected to the equatorial bearing ring by screws, as is the bearing frame. The motherboard is installed on the bearing frame. Antennas are installed on the outer surface of the equatorial bearing ring. Electronic devices are distributed on the bearing frame and the motherboard. The origin of the satellite body coordinate system is defined as the center of sphere. The $Y$-axis is perpendicular to the equatorial bearing ring and the positive direction points to the upper hemisphere. The $Z$-axis points in the opposite direction of the GNSS antenna. The $X$-axis has a right-handed spiral relationship with the $Y$-axis and the $Z$-axis.

The main devices of Q-SAT are shown in Figure 2. Attitude control computer (ACC), on-board computer (OBC), ISM transceiver, and single-frequency GNSS receiver (S-F GNSS) are installed on the motherboard. USB transceiver, flywheel, microwave amplifier (MA), batteries, power control unit (PCU), and the main payload dual-frequency GNSS receiver (D-F GNSS) are fixed on the bearing frame. Another payload, the panoramic camera together with the on-board intelligent processing module are installed on the equatorial bearing ring. The camera lens is exposed outside the satellite.

2.2. Thermal Control Based on Integrated Spherical Structure. Qualitative discussion about thermal control is carried out 
TABLE 2: The reference material parameters in the simulation.

\begin{tabular}{lcccc}
\hline Material & $\begin{array}{c}\text { Coefficient of thermal conductivity } \\
(\mathrm{W} / \mathrm{m} \cdot \mathrm{K})\end{array}$ & $\begin{array}{c}\text { Specific heat capacity }(\mathrm{J} / \\
\mathrm{kg} \cdot \mathrm{K})\end{array}$ & $\begin{array}{c}\text { Solar radiation } \\
\text { absorption rate }\end{array}$ & $\begin{array}{c}\text { Infrared } \\
\text { emissivity }\end{array}$ \\
\hline Black anodized Al alloy & 121.4 & 920.9 & 0.90 & 0.85 \\
Ecru conductive oxidation & 121.4 & 920.9 & 0.16 & 0.2 \\
Al alloy & - & - & 0.92 & 0.82 \\
Solar cell & - & - & 0.42 & 0.25 \\
Polyimide & 390 & 390 & 0.04 \\
Gold-plated copper & & & 0.89 & 0.25 \\
\hline
\end{tabular}

TABLE 3: The powers of devices in WHC and WCC.

\begin{tabular}{lcc}
\hline Device & Power in WHC (W) & Power in WCC (W) \\
\hline PMU and batteries & 1.0 & 1.0 \\
OBC & 0.8 & 0.8 \\
ACC & 0.8 & 0.8 \\
Motherboard & 1.0 & 1.0 \\
USB & 3.6 & 3.6 \\
D-F GNSS & 2.0 & 2.0 \\
Flywheel & 6.0 & 1.0 \\
ISM & 2.1 & 0.1 \\
S-F GNSS & 1.5 & 1.5 \\
Microwave amplifier & 7.0 & 0.0 \\
Camera & 4.0 & 0.1 \\
\hline
\end{tabular}

before numerical simulation calculations. Since space is a vacuum environment, satellites can only interact with the environment through radiation. Therefore, for Q-SAT, thermal interaction includes three ways: (1) direct solar radiation, (2) solar radiation reflected from the Earth (albedo radiation) and direct infrared radiation from the Earth, and (3) the energy of heat radiation from the outer surface of the spacecraft to deep space. Due to the small magnitude, the thermal effects of other planets can be ignored in engineering. When the heat obtained by the thermal interaction between the satellite and the environment is equal to the internal heat dissipation, the spacecraft is in a state of thermal equilibrium [19]. The orbit and sunlight condition are shown in Figure 3. According to mission, Q-SAT chose a $500 \mathrm{~km}$ round high sun-synchronous orbit (SSO). During the flight, the Q-SAT maintains the attitude of a 3-axis stable pointing to the Earth. The $-Z$-axis always points to the center of the Earth.

The heat absorbed by Q-SAT is directly proportional to the irradiated area according to the law of radiation. The orbit of Q-SAT is SSO, which is selected by many small satellites. Satellites with similar shapes, such as common cuboid satellites, have a similar area-to-mass ratio. However, Q-SAT is different. As mentioned above, the purpose of spherical design for Q-SAT is to ensure a constant area-to-mass ratio. In order to make the effect of atmospheric drag more obvious, the altitude of Q-SAT should drop faster. For the above reason, the area-to-mass ratio of Q-SAT is much larger than that of a normal satellite. To put it vividly, the interior of QSAT is empty compared to most satellites. The area-to-mass ratio of Q-SAT is $0.00965 \mathrm{~m}^{2} / \mathrm{kg}$, which is about 10 times larger than common satellites. Q-SAT is very easy to heat up under the heat radiation in space compared to other small satellites in SSO because of larger exposed area and smaller mass (the main materials of satellites are usually similar, such as aluminum alloy, so the specific heat capacity is relatively close). Therefore, overheating is the main challenge for thermal control according to preliminary analysis. Of course, the above argument is only a preliminary trend estimate, and subsequent numerical simulation should be used to confirm the reliability of the thermal design.

From Figure 3, heat radiation input and output surface of Q-SAT can be seen obviously. The hemisphere in the $Y$-direction is always heated during the illumination period, most is radiated by sun, and a part is heated by Earth reflection. Part of the hemisphere in the $+Y$-direction is heated by sunlight and Earth albedo radiation. The heat output surface is another part of the hemisphere in the $+Y$-direction. According to thermal balance conditions, this is the only heat dissipation area. Therefore, it is necessary to increase the heat exchange between the $+Y$ hemisphere and the rest as much as possible in order to prevent the satellite from overheating. The challenge is to use limited passive thermal control methods to achieve directional heat transfer. Both the solar radiant heat in the $-Y$ hemisphere and the heat produced by the devices inside Q-SAT need to be transferred to the $+Y$ hemisphere in time. An ingenious thermal control method by using thermal isolation layer and high emissivity multilayer is proposed to solve this problem.

Figure 4 is the overview of Q-SAT thermal design. The satellite is divided into 5 thermal modules including $+Y$ hemisphere, $-Y$ hemisphere, equatorial ring, polyimide insulation, and the core (bearing frame, motherboard with devices on them) according the structure layout. The $\pm Y$ hemispheres are connected by the equatorial ring. The core is connected to the equatorial ring by polyimide insulation. The thermal resistance between the 3 modules in the outer layer is designed small so that the heat absorbed from the thermal radiation can be conducted to the $+Y$ hemisphere quickly to complete the heat dissipation. Polyimide is used to insulate the high temperature of the $-Y$ hemisphere directly exposed to the sun radiation. Multilayers are attached to the inner surface of the $+Y$ hemisphere to ensure the heat exchange between the core and the $+Y$ hemisphere 


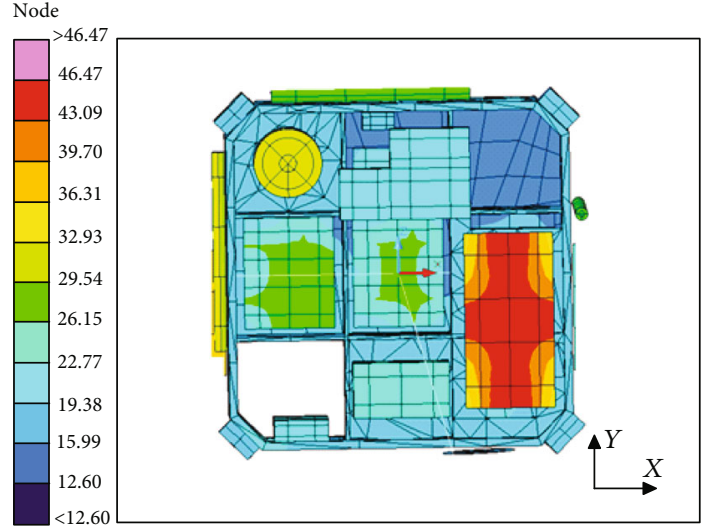

Temperature $\left({ }^{\circ} \mathrm{C}\right)$

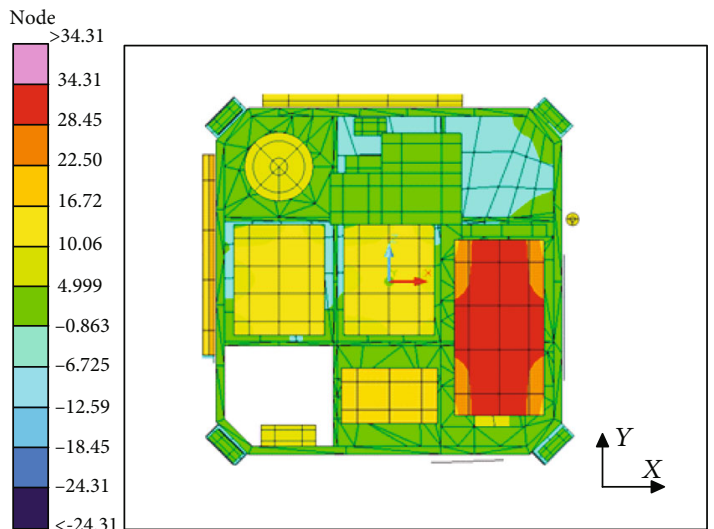

Temperature $\left({ }^{\circ} \mathrm{C}\right)$

WHC

WCC

Figure 7: The core temperature distribution of thermal analysis.

TABle 4: Temperatures of devices inside Q-SAT.

\begin{tabular}{lcccc}
\hline Device & $\begin{array}{c}\text { Allowing } \\
\text { range } \\
\left({ }^{\circ} \mathrm{C}\right)\end{array}$ & $\begin{array}{c}\text { Analysis } \\
\text { result in } \\
\text { WHC }\left({ }^{\circ} \mathrm{C}\right)\end{array}$ & $\begin{array}{c}\text { Analysis } \\
\text { result in } \\
\text { WCC }\left({ }^{\circ} \mathrm{C}\right)\end{array}$ & $\begin{array}{c}\text { Test } \\
\text { result } \\
\left({ }^{\circ} \mathrm{C}\right)\end{array}$ \\
\hline PCU & $-10 \sim+50$ & $21 \sim 23$ & $15 \sim 21$ & 20.4 \\
Batteries & $0 \sim+40$ & $15 \sim 21$ & $9 \sim 11$ & 22.7 \\
OBC & $-40 \sim+85$ & $13 \sim 27$ & $3 \sim 17$ & 18.1 \\
ACC & $-40 \sim+85$ & $13 \sim 27$ & $3 \sim 17$ & 15.8 \\
Motherboard & $-40 \sim+85$ & $8 \sim 21$ & $-3 \sim 10$ & 12.5 \\
USB & $-20 \sim+65$ & $32 \sim 47$ & $20 \sim 35$ & 36.9 \\
D-F GNSS & $-25 \sim+55$ & $18 \sim 24$ & $6 \sim 11$ & 14.1 \\
Magnetometer & $-40 \sim+85$ & $15 \sim 20$ & $3 \sim 7$ & 11.0 \\
Flywheel & $-30 \sim+60$ & $32 \sim 33$ & $7 \sim 9$ & 11.3 \\
ISM & $-30 \sim+60$ & $10 \sim 29$ & $-2 \sim 3$ & 20.3 \\
IMU & $-30 \sim+50$ & $16 \sim 22$ & $6 \sim 10$ & 21.3 \\
S-F GNSS & $-25 \sim+50$ & $12 \sim 22$ & $0 \sim 9$ & 13.4 \\
MA & $-25 \sim+50$ & $16 \sim 25$ & $2 \sim 6$ & 17.0 \\
\hline
\end{tabular}

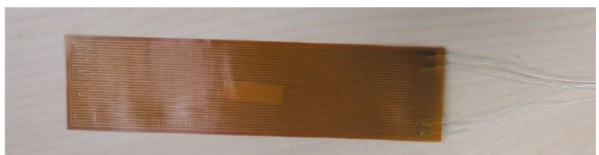

FIgURE 8: The electric heater of polyimide film type.

and the heat generated by the devices can be transferred in time. On the contrary, the inner surface of $-Y$ hemisphere is aluminum alloy of conductive oxidation; the infrared emission rate of which is very low. The core ability of heat transfer by radiation is very low. This design reduces the heat exchange capability between the $-Y$ hemisphere and the core through radiation.

The hemisphere is specially designed in order to achieve thermal equilibrium quickly. The hemisphere includes frame and solar panels (Figure 5). The hemisphere frame is formed by a single piece of aluminum alloy instead of assembly to ensure the low thermal resistance. The surface of the frame is anodized to ensure emissivity. The solar cells are pasted on the panel through polyimide film. It can be seen from the figures that most of the outer surface of the hemisphere is occupied by the panels. The area and emissivity of solar cells and polyimide can be accurately calculated, so the heat radiation characteristics of the hemisphere can be modeled accurately. Equatorial ring also uses aluminum alloy. Silica gel is applied to the contact surface between hemispheres and the equatorial ring to improve the thermal conductivity.

The main devices are installed on the $-Y$ side of the core to ensure heat are radiated to the cooling surface of Q-SAT. These devices and the bearing frame adopt an installation method that enhances heat conduction. These devices are installed on the bearing frame through thermally conductive materials. The structure surfaces are black anodized to provide reliable emissivity and absorptivity. Active heating devices are used on the lithium batteries to prevent it from working at low temperatures.

2.3. Simulation. A thermal analysis of Q-SAT is created in the simulation software Thermal Desktop (TD). Satellite's heat input conditions are created based on the planned orbit. The key parameters of heat input include the angle between solar radiation and orbit $\beta$ (shown in Figure 3), the solar constant, and the heat generated by the devices inside QSAT. Changes in these parameters will result in the worsthot case (WHC) and the worst-cold case (WCC). When the angle $\beta$ is the largest, the solar radiation reaches the $Y$ hemisphere the least. Considering the contact thermal resistance between the two hemispheres, the $+Y$ hemisphere accumulates the most heat at this time, which is the WHC. In the same way, when the angle $\beta$ is the smallest, the satellite is in WCC. WHC and WCC are given in Table 1. The value of the solar constant considers the fluctuation range of solar radiation. The thermal design should ensure that the operating temperatures of all devices are within the permitted range under different cases. 


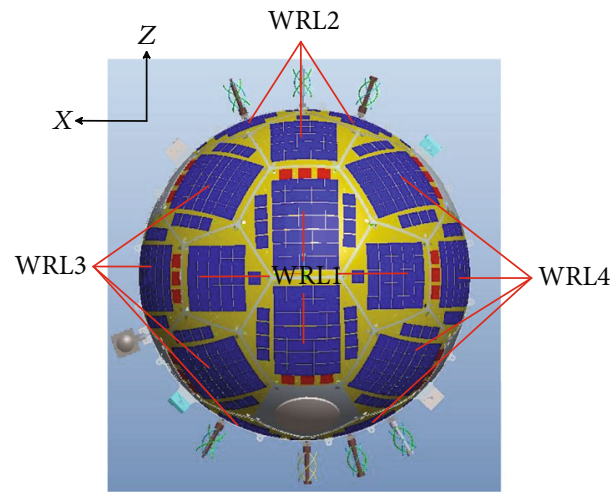

+ Y Hemisphere

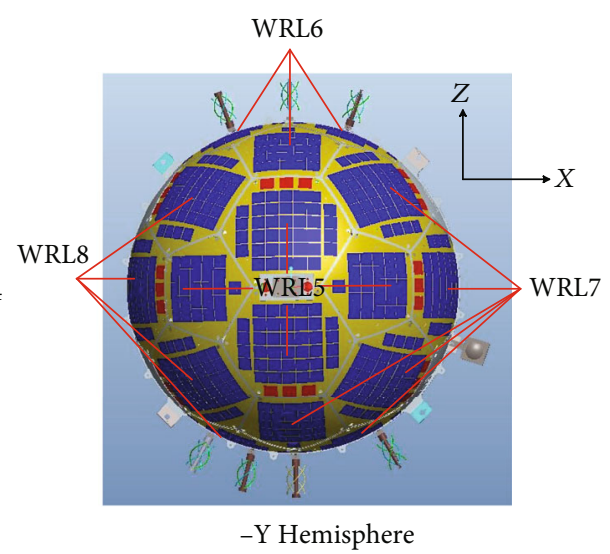

-Y Hemisphere

Figure 9: The layout of the distributed heaters.

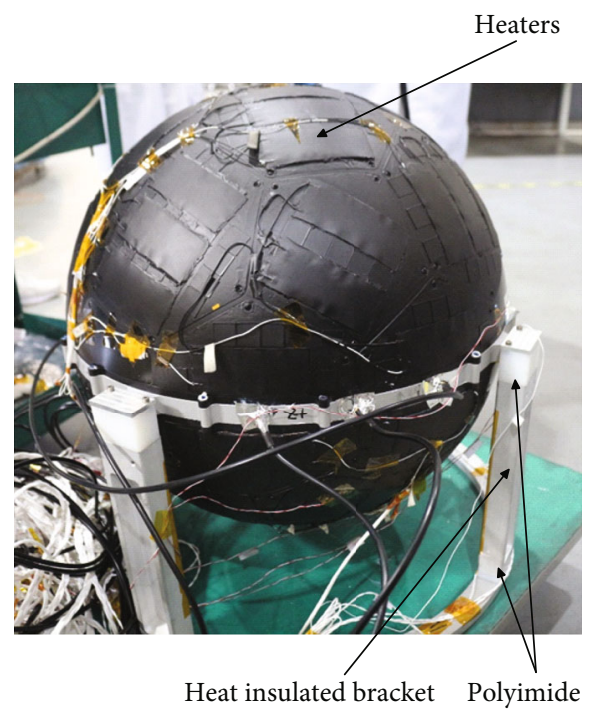

FIgURE 10: Q-SAT before thermal test.

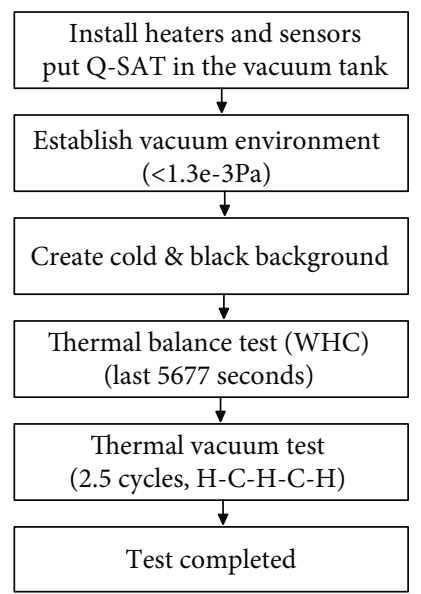

Figure 11: Steps of thermal test (H: hot; C: cold).
A thermal model of Q-SAT is established with simplification on the basis of the real model, while the thermal capacity of each component is kept unchanged. The model is shown in Figure 6. According to the parameters in Table 1, a transient thermal analysis is performed on the satellite, and all passive thermal control measures, the active heating control on the batteries, and external thermal radiation were considered in the calculation. Material parameters mainly used in the simulation are shown in Table 2 . The powers of devices under the two cases are listed in Table 3. Layout of the devices are shown in Figure 2.

Figure 7 shows the core temperature distribution of thermal analysis in WHC and WCC. Table 4 reports the simulation results, including the temperature variation range of each component under WHC and WCC, together with the allowable temperature range as a comparison. The time to reach thermal equilibrium is $83640 \mathrm{~s}$ in WHC and $52520 \mathrm{~s}$ in WCC. All the working temperatures of the devices are in the allowable range, according to the analysis results of graphics and tables of statistics, whether in WHC or WCC. Thermal analysis results show that the thermal control design can effectively control the temperature of Q-SAT in a reasonable range. Furthermore, the thermal test is necessary to verify the reliability of thermal design.

\section{Thermal Test}

3.1. Distributed Heat Flow Method Based on Electric Heaters. Thermal test is the basis for judging the reliability of thermal design. According to the previous analysis, Q-SAT is more concerned about overheating. Therefore, the thermal test strategy based on WHC case was selected for saving time and cost. The philosophy of thermal test is to verify the core of Q-SAT as a whole instead of testing each device one by one. The electric heaters are mounted on the surface of the satellite to accurately simulate the difference in radiation at different positions on the spherical surface.

There are additional problems with the use of the heaters for thermal testing of Q-SAT. Heaters must not directly contact the solar cells. A pair of hemispheres together with polyimide and simulated solar cells is made to solve this 


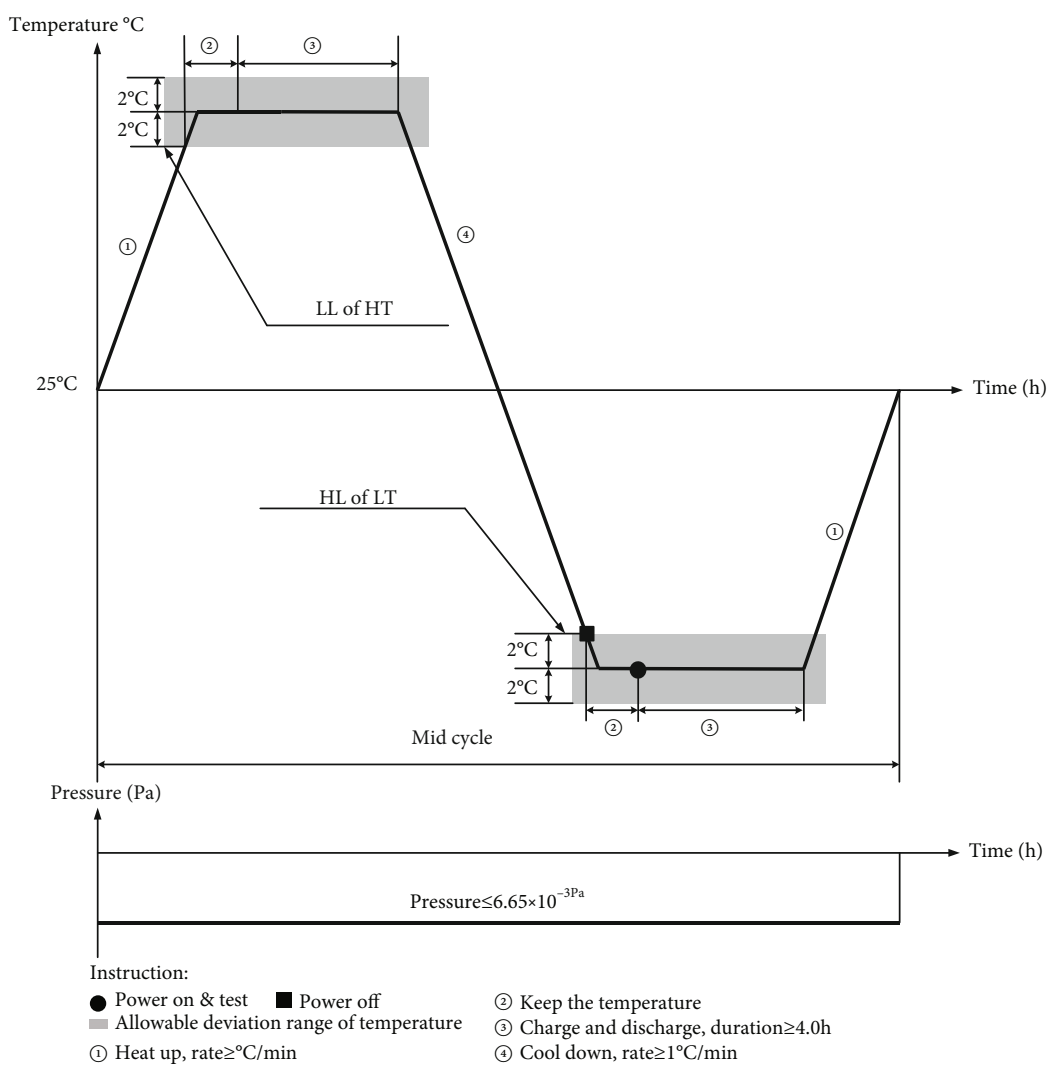

(a) The first and the lase cycle of thermal vacuum test

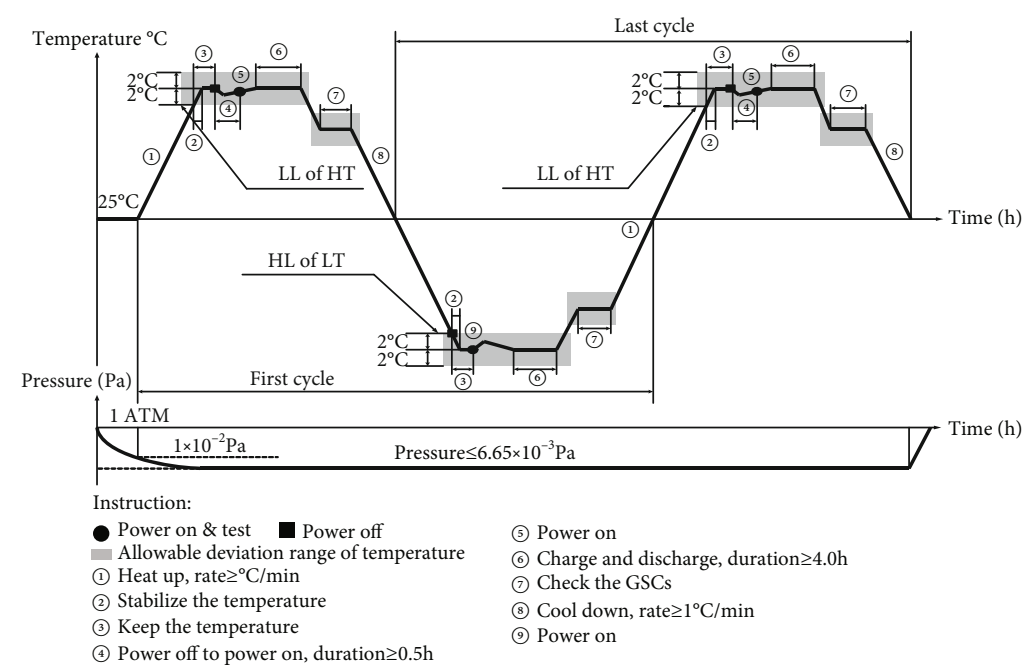

(b) The middle cycle of the thermal vacuum test

FIgURE 12: Temperature curve of thermal vacuum test.

problem. They are used to replace the real hemisphere structures and solar cells in thermal tests to facilitate surface mounting of heaters. The difference between the two sets of hemispheres is whether the real solar cells are used. Solar cells are mature aerospace products with sufficient flight experience, so it is reasonable to exclude them from test. This test method is to use distributed flexible electric heaters (Figure 8) attached to the surface of the sphere to heat the satellite according to analyzed power. Unlike a plane, the incident angles of heat radiation at different positions on the spherical surface at the same time are different. Because only a limited number of discrete heaters can be mounted on the sphere, the power of each heater should be the integral of the corresponding area's radiation input density. Each solar panel is set as an independent heating unit, and 1 3 heaters are attached on it according to the area of the panel.

The layout scheme of the distributed external heaters is shown in Figure 9. There are 8 heating circuits 


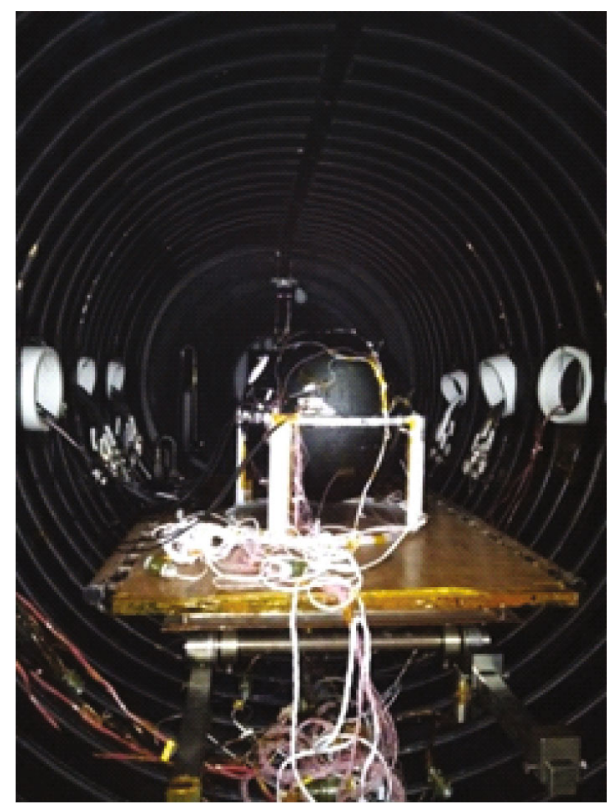

Figure 13: Thermal test scene.

Table 5: Parameters of the heating loops.

\begin{tabular}{ccccc}
\hline Loop & $\begin{array}{c}\text { Load } \\
(\Omega)\end{array}$ & $\begin{array}{c}\text { Heat area } \\
\left(\mathrm{mm}^{2}\right)\end{array}$ & $\begin{array}{c}\text { Power }(\mathrm{W}) \\
(0 \sim 1893 \mathrm{~s})\end{array}$ & $\begin{array}{c}\text { Power }(\mathrm{W}) \\
(1893 \sim 5677 \mathrm{~s})\end{array}$ \\
\hline WRL1 & 100 & $40 \times 100 \times 10$ & 4.71 & 9.04 \\
WRL2 & 40 & $40 \times 100 \times 4$ & 5.42 & 10.66 \\
WRL3 & 100 & $40 \times 100 \times 10$ & 5.12 & 26.54 \\
WRL4 & 100 & $40 \times 100 \times 10$ & 5.18 & 26.59 \\
WRL5 & 100 & $40 \times 100 \times 10$ & 4.76 & 47.14 \\
WRL6 & 40 & $40 \times 100 \times 4$ & 5.42 & 14.08 \\
WRL7 & 120 & $40 \times 100 \times 12$ & 5.16 & 59.18 \\
WRL8 & 100 & $40 \times 100 \times 10$ & 5.10 & 49.13 \\
\hline
\end{tabular}

(WRL1 WRL8) in the thermal, and several heaters are in series in each heating loop. The heat input density of the panels belonging to the same loop is the same, because the average integrated radiation of these panels during an orbital period is approximately the same. The heaters cover all the solar panels on the two hemispheres.

After the heaters are attached on the test hemispheres, the surface of Q-SAT is sprayed with a kind of special black paint to eliminate the environmental influence of the thermal vacuum tank. A special heat-insulated bracket is also designed to isolate the heat input from the environment, with polyimide structures deployed at its junctions. Figure 10 is the overview of Q-SAT before thermal test.

Figure 11 lists the steps throughout the test. The thermal balance test is conducted after the test environment is established. Only WHC is simulated as the heat input because the time is quite tight; besides, overheat may be a destructive problem, and the active heater onboard Q-SAT can prevent the satellite from too cold. The ambient temperature control curve of thermal vacuum test is shown in Figure 12.

3.2. Results of Thermal Balance Test. The thermal test (Figure 13) was conducted according to the method above. The parameters of the heating loops are listed in Table 5. The loops WRL5, WRL7, and WRL8 have obviously larger powers, which is consistent with the $-Y$ hemisphere being the main heated part on the orbit. The first 1893s of thermal balance test is to simulate the state in shadow period while the rest time is illumination period. The power of each heater is the average value in the orbital period. The correctness of thermal design and analysis can be validated through thermal balance test.

Temperature sensors (thermistor MF501) were installed near each device before test. The stable temperatures of devices when the satellite is thermal balanced, as the results, are shown in Table 4 . The test results are in good agreement with the simulation results (WHC), indicating that the thermal design and analysis have a high credibility and reliability. Therefore, it is reasonable to believe that the analysis results in WCC are also reliable, although the corresponding thermal balance test has not been carried out due to short time.

The main purpose of the thermal vacuum test is to verify the environmental adaptability of devices and structure. Heat input in the thermal vacuum test is adjusted based on the parameters in Table 5. Examination on Q-SAT before and after the thermal vacuum test showed that its performance has not changed.

\section{On-Orbit Performance}

Q-SAT was launched on August 6, 2020, atop the CZ-2D rocket from Jiuquan Satellite Center, northwest China, and it works well on orbit now. The telemetry data contains the temperature information collected by the thermistor on the satellite. This section introduces the thermal state of satellite's daily orbit operation on October 22, 2020, to verify the thermal design. The orbit period is about 90 minutes, and $\beta$ is $20.8^{\circ}$ on this day. The case of this day is between WHC and WCC. Thermistors on the inner surface of the hemispheres (Figure 14) detect the exposure of Q-SAT.

The temperatures of the hemispheres are recorded in Figure 15 (WHC) and Figure 16 (WCC). The period of surface temperature change is approximately 90 minutes, which is the same as the period of the satellite orbit. The hottest panel (10\#) is about $55^{\circ} \mathrm{C}$, and the opposite position (02\#) is the coldest at $-8^{\circ} \mathrm{C}$ in WHC. Considering that there is a $5 \mathrm{~mm}$ thick aluminum alloy between the sensor and the outer surface of the satellite, the measurement result may have a deviation of $3 \sim 5^{\circ} \mathrm{C}$. The max temperature difference on the satellite surface is about $50 \sim 60^{\circ} \mathrm{C}$ according to above facts. This temperature difference shows that the design of using an integrated spherical structure for thermal control is very reasonable. The temperatures of the neighboring panels distributed on opposite sides of the equatorial ring are very close, which indicates that the thermal resistance between the structures is well controlled. When the satellite 


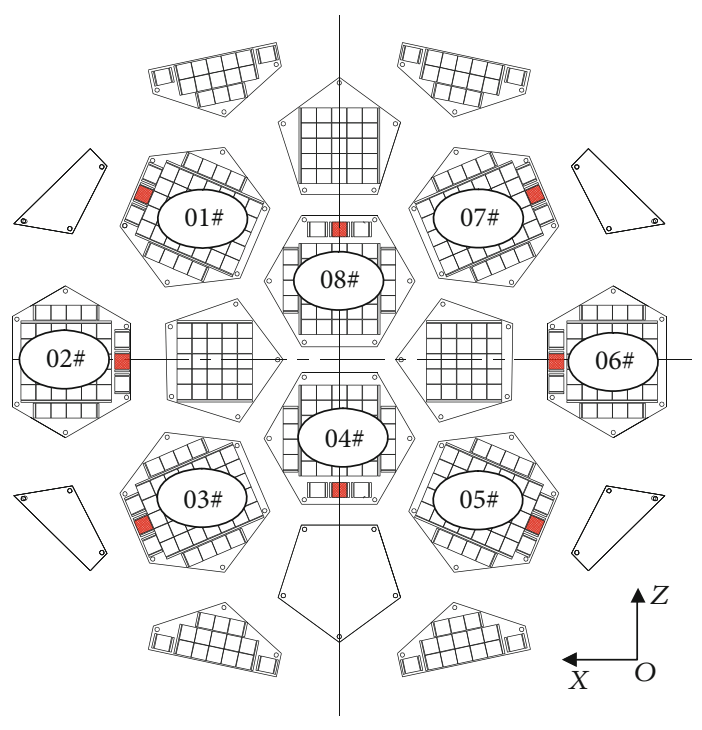

+ Y Hemisphere

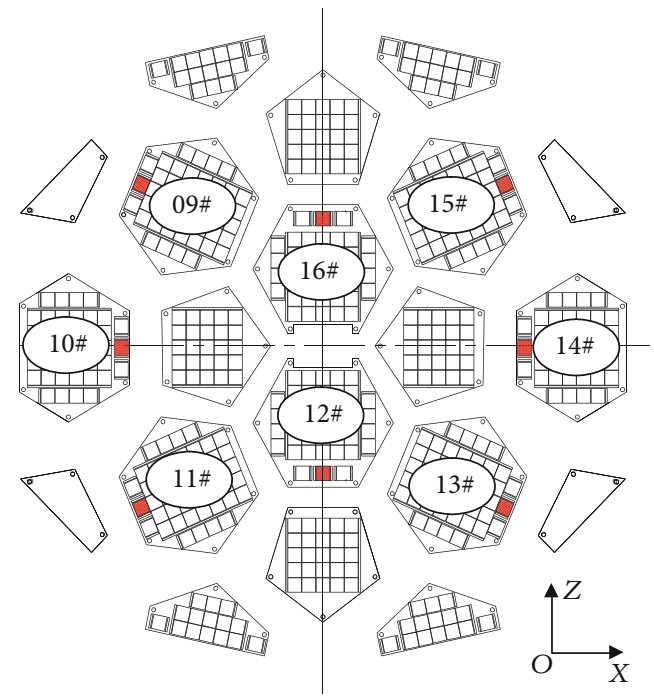

-Y Hemisphere

FIGURE 14: Thermistors on the hemispheres with numbers.

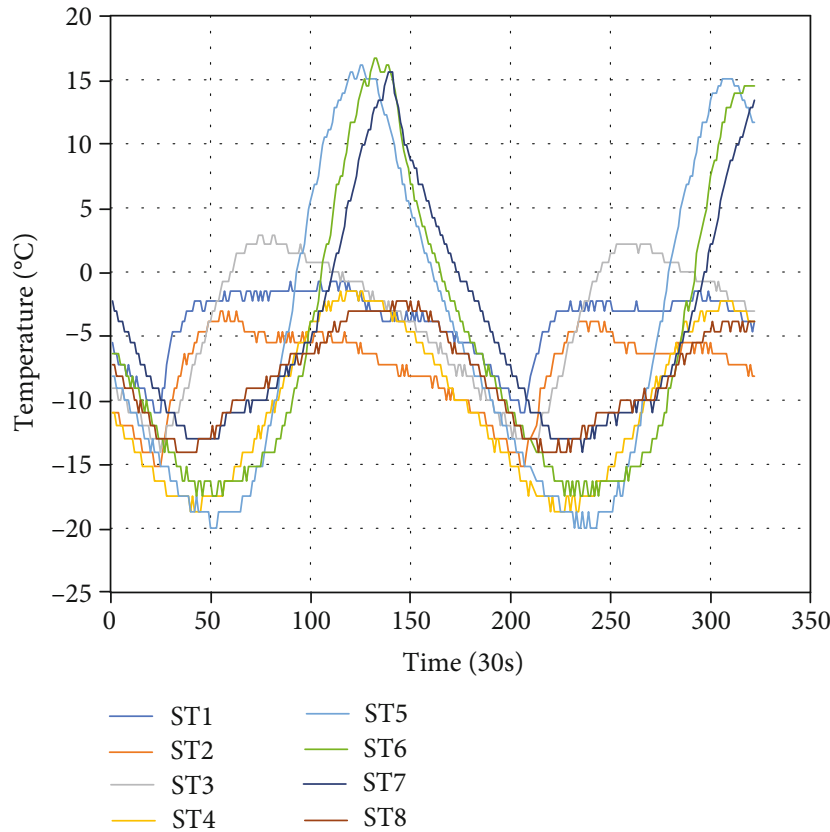

(a) Temperatures of $+Y$ hemisphere

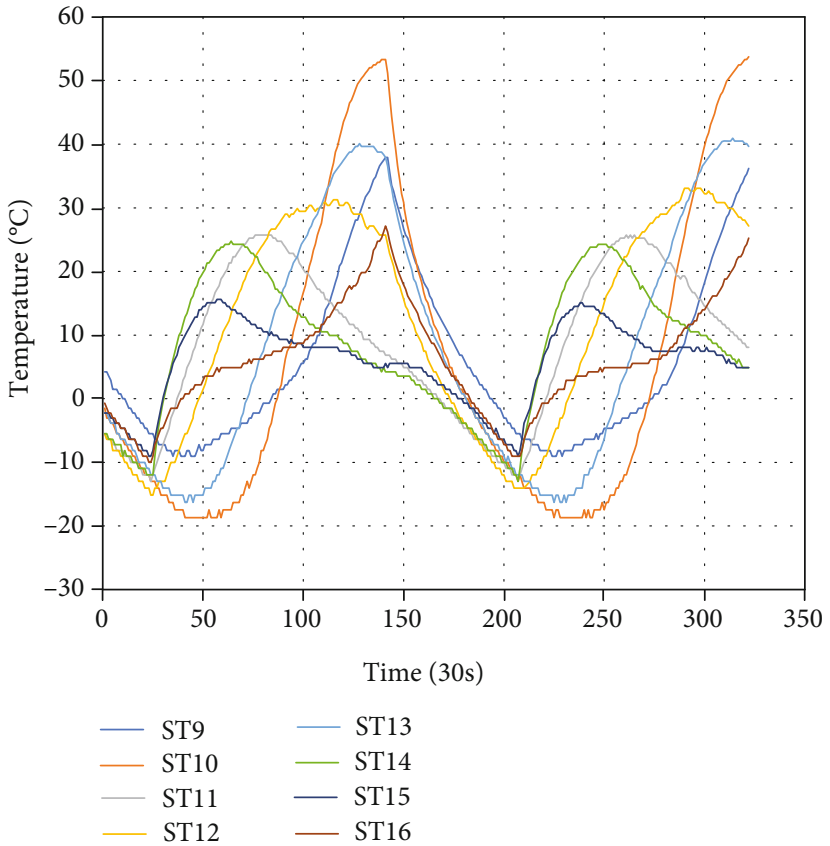

(b) Temperatures of $-Y$ hemisphere

FIgURE 15: Temperatures of hemispheres on orbit on 2021.2.10 (X: time (30 s); Y: temperature $\left({ }^{\circ} \mathrm{C}\right)$; ST N: surface temperature of $N \#$ panel in Figure 14).

is in WCC, the temperature of the spherical surface is about $-20^{\circ} \mathrm{C}$.

The telemetry data of satellite's internal thermistors is plotted in Figure 17 (WHC) and Figure 18 (WCC). It can be seen from the curves that the temperatures inside QSAT also fluctuates in the orbital period. The temperature of all devices is maintained at $0 \sim 20^{\circ} \mathrm{C}$, and the batteries are in $9 \sim 19^{\circ} \mathrm{C}$, which is a quite perfect range. The temperature of some devices is lower than expected. This is because the operating power of these devices has been adjusted to a certain extent when running on orbit. The daily remote temperature data is similar to the one shown in this section. The temperature control system successfully guaranteed the daily operation of Q-SAT. 


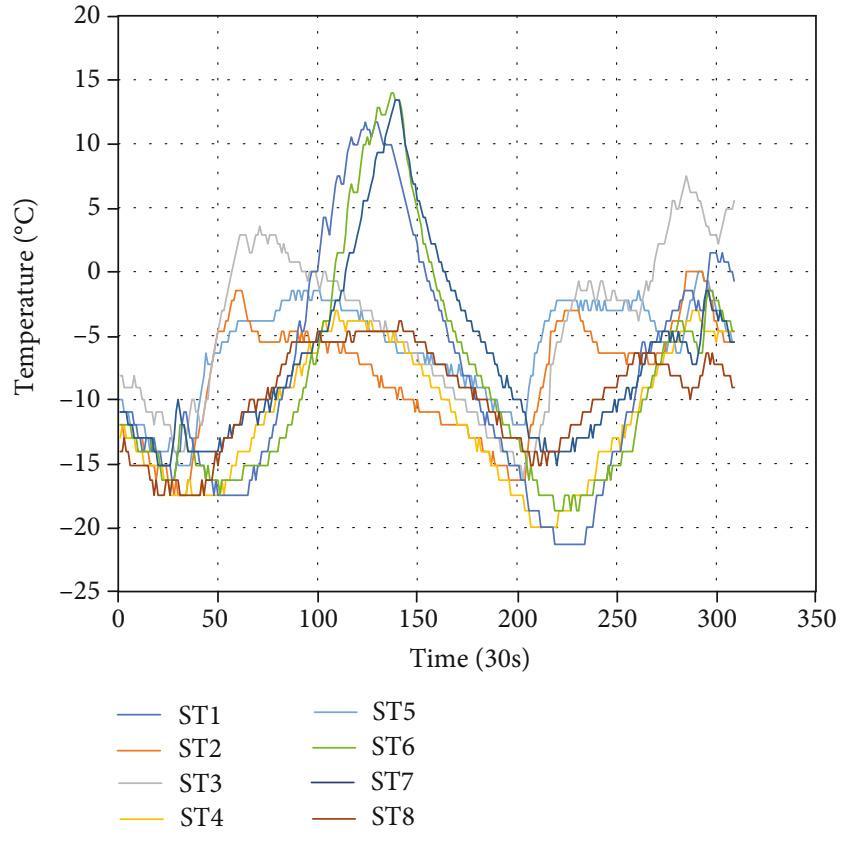

(a) Temperatures of $+Y$ hemisphere

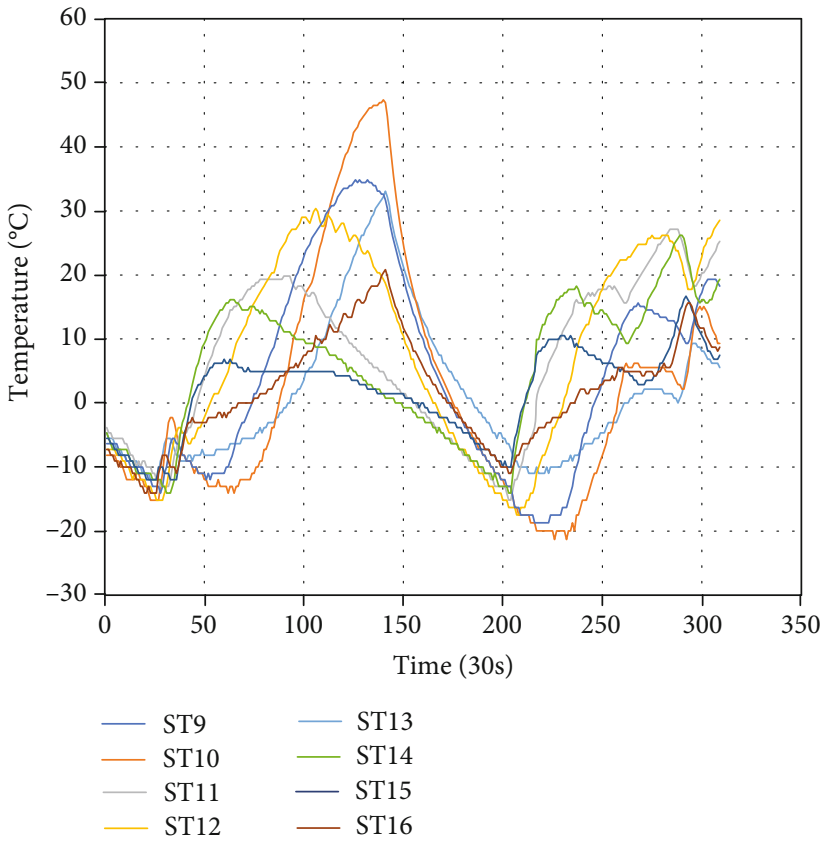

(b) Temperatures of $-Y$ hemisphere

Figure 16: Temperatures of hemispheres on orbit on 2021.6 .03 (X: time (30 s); $Y$ : temperature $\left({ }^{\circ} \mathrm{C}\right)$; ST $N$ : surface temperature of $N \#$ panel in Figure 14).

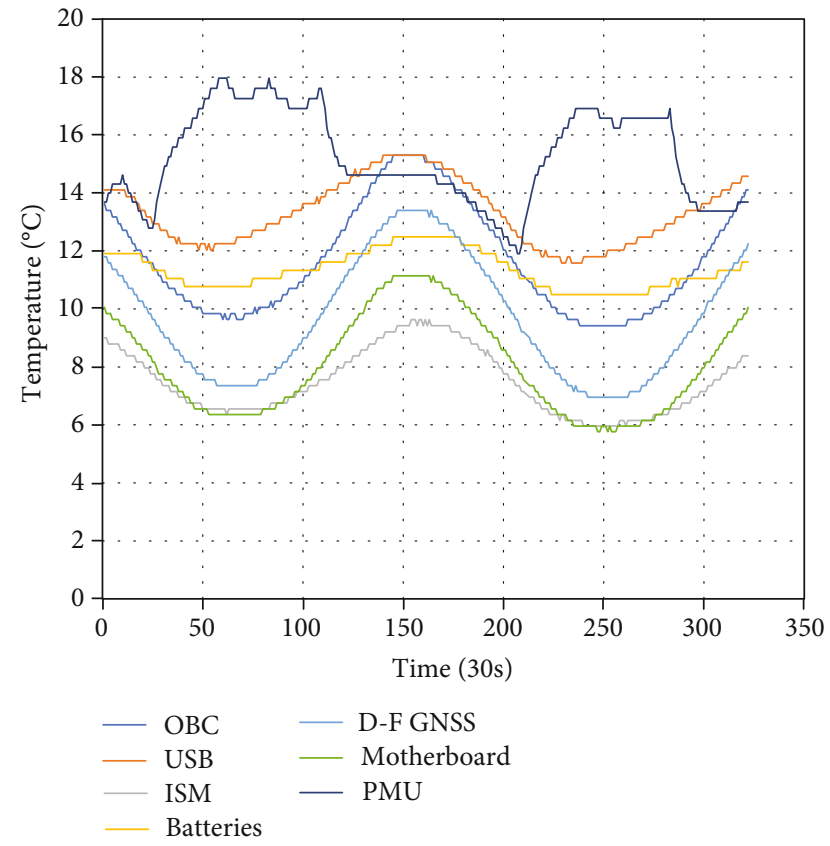

FIgURE 17: Temperatures of devices inside Q-SAT on 2021.2.10.

\section{Conclusion}

According to the requirement of Q-SAT, a passive thermal control system based on integrated spherical structure is proposed. Satellite's on-orbit heat input is analyzed, and the worst conditions are determined. The idea of the thermal design is to transfer the heat from the exposed surface and

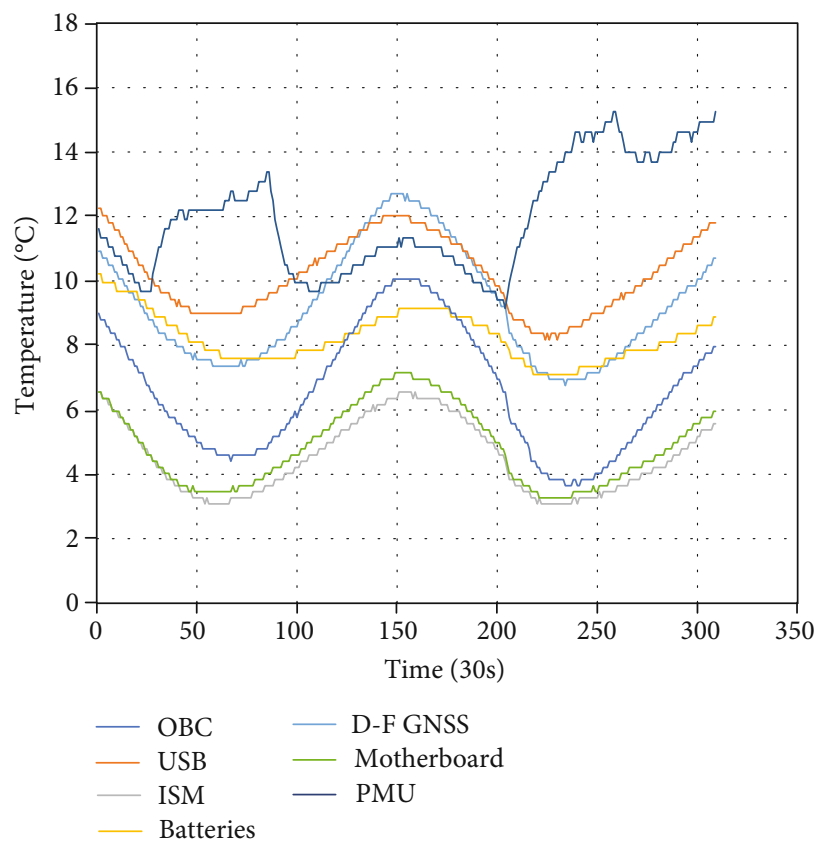

Figure 18: Temperatures of devices inside Q-SAT on 2021.6.03.

the devices in Q-SAT to the heat dissipation surface as soon as possible. The main method of thermal control is to use thermal insulation materials, thermal conductive materials, and thermal control coatings to control the path of heat exchange. Analysis shows that this method is feasible.

A thermal test method based on distributed electric heaters is used to adapt the spherical structure of Q-SAT. 
External heat flow input is accurately simulated. The test results are consistent with the simulation results. After the satellite is launched, the telemetry data shows that its internal temperature is controlled very well. The excellent operation indicates that thermal design of Q-SAT is satisfactory. Relying on reasonable thermal design and accurate simulation, the passive thermal control system based on COTS can meet the requirements of small satellites. With the vigorous development of small satellite technology, space science exploration missions may have more special-shaped satellites. The work in this paper can provide reference for more small satellite engineering practice.

\section{Data Availability}

The data used to support the findings of this study is included within the article and is also available from the corresponding author upon request.

\section{Conflicts of Interest}

The authors declare that they have no conflicts of interest.

\section{Acknowledgments}

The authors thank Zhang Qi and Cai Yingkai (Ph.D students) for their assistance in processing on-orbit telemetry data. The authors also thank Fan Hongmei from the Shandong Aerospace Electronics Institute for her advice on thermal design and test. Funding for this paper was provided from the Key Research and Development Projects in Guangdong Province, China (No. 2020B090919002).

\section{References}

[1] Y. Zhao, Z. Wang, and Y. Zhang, "A spherical micro satellite design and detection method for upper atmospheric density estimation," International Journal of Aerospace Engineering, vol. 2019, Article ID 1758956, 15 pages, 2019.

[2] D. Park, K. Miyata, and H. Nagano, "Thermal design and validation of radiation detector for the ChubuSat-2 microsatellite with high-thermal-conductive graphite sheets," Acta Astronautica, vol. 136, pp. 387-394, 2017.

[3] A. Ali, J. Tong, H. Ali, M. R. Mughal, and L. M. Reyneri, “A detailed thermal and effective induced residual spin rate analysis for LEO small satellites," IEEE Access, vol. 8, pp. 146196146207, 2020.

[4] V. Baturkin, "Micro-satellites thermal control-concepts and components," Acta Astronautica, vol. 56, no. 1-2, pp. 161170, 2005.

[5] W. Guo, Y. Li, Y. Z. Li et al., "A self-driven temperature and flow rate co-adjustment mechanism based on ShapeMemory-Alloy (SMA) assembly for an adaptive thermal control coldplate module with on-orbit service characteristics," Applied Thermal Engineering, vol. 114, pp. 744-755, 2017.

[6] P. R. Mashaei and M. Shahryari, "Effect of nanofluid on thermal performance of heat pipe with two evaporators; application to satellite equipment cooling," Acta Astronautica, vol. 111, pp. 345-355, 2015.

[7] A. Ali, K. Ullah, H. Rehman, I. Bari, and L. M. Reyneri, "Thermal characterisation analysis and modelling techniques for
CubeSat-sized spacecrafts," The Aeronautical Journal, vol. 121, no. 1246, pp. 1858-1878, 2017.

[8] D. Gilmore, Ed., Satellite Thermal Control Handbook, The Aerospace Corporation Press, El Segundo, CA, 2002.

[9] C. Latham, "Technical brief, thermal resistance of interface materials as a function of pressure," Journal Electronics Cooling, vol. 2, no. 3, p. 35, 1996.

[10] Y. Shimakawa, T. Yoshitake, Y. Kubo et al., "A variableemittance radiator based on a metal-insulator transition of (La,Sr)MnO3 thin films," Applied Physics Letters, vol. 80, no. 25, pp. 4864-4866, 2002.

[11] M. Bonnici, P. Mollicone, M. Fenech, and M. Azzopardi, "Analytical and numerical models for thermal related design of a new pico-satellite," Applied Thermal Engineering, vol. 159, article 113908, 2019.

[12] J. Berk, J. Straub, and D. Whalen, "The open prototype for educational nanosats: fixing the other side of the small satellite cost equation," in 2013 IEEE Aerospace Conference, Big Sky, MT, USA, 2013.

[13] R. Kovács and V. Józsa, "Thermal analysis of the SMOG-1 PocketQube satellite," Applied Thermal Engineering, vol. 139, pp. 506-513, 2018.

[14] I. Grande, A. Sanz-Andrs, C. Guerra, and G. Alonso, "Analytical study of the thermal behavior and stability of a small satellite," Applied Thermal Engineering, vol. 29, pp. 2567-2573, 2009.

[15] M. Bulut and N. Sozbir, "Analytical investigation of a nanosatellite panel surface temperatures for different altitudes and panel combinations," Applied Thermal Engineering, vol. 75, pp. 1076-1083, 2015.

[16] E. Escobar, M. Diaz, and J. Zagal, "Evolutionary design of a satellite thermal control system: real experiments for a CubeSat mission," Applied Thermal Engineering, vol. 105, pp. 490500, 2016.

[17] X. Jin, "Mechanical environmental test for artificial satellites," Chinese Spaceflight, vol. 8, pp. 17-21, 1993.

[18] J. S. Almeida, M. B. Santos, D. L. Panissi, and E. C. Garcia, "Effectiveness of low-cost thermal vacuum tests of a micro-satellite," Acta Astronautica, vol. 59, no. 6, pp. 483-489, 2006.

[19] A. Farrahi and I. Pérez-Grande, "Simplified analysis of the thermal behavior of a spinning satellite flying over sunsynchronous orbits," Applied Thermal Engineering, vol. 125, pp. 1146-1156, 2017. 